\title{
BEAM-INDUCED ENERGY DEPOSITION IN MUON STORAGE RINGS
}

\author{
N. V. Mokhov ${ }^{\dagger}$, C. J. Johnstone, FNAL, Batavia, IL 60510, USA \\ B. L. Parker, BNL, Upton, NY 11973, USA
}

\begin{abstract}
Beam-induced radiation effects have been simulated for 20 and $50 \mathrm{GeV}$ muon storage rings designed for a Neutrino Factory. It is shown that by appropriately shielding the superconducting magnets, quench stability, acceptable dynamic heat loads, and low residual dose rates can be achieved. Alternatively, if a specially-designed skew focusing magnet without superconducting coils on the magnet's mid-plane is used, then the energy is deposited preferentially in the warm iron yoke or outer cryostat layers and internal shielding may not be required. In addition to the component irradiation analysis, shielding studies have been performed. Calculations of the external radiation were done for both designs but the internal energy deposition calculations for the $20 \mathrm{GeV}$ Study-2 lattice are still in progress.
\end{abstract}

\section{MUON RINGS}

The energy deposition from beam loss and muon decay has been simulated for $50-\mathrm{GeV}$ Study-1 [1] and 20GeV Study-2 [2] muon storage rings with the MARS 14 code [3]. The rings are similar: a racetrack geometry with long, normal-conducting magnets in production straights and short, superconducting (SC) ones in the arcs. The magnets were profiled in detail for the simulations. From these calculations, prompt and residual radiation levels have been determined in the magnets, in the vicinity of the tunnel and at large distances from the complex. In Study-1 case, we follow results of Ref. [4].

The Study-1 parameters and radiation requirements for a $50-\mathrm{GeV}$ muon beam were taken from [1]. Lattice functions are shown in Fig. 1. The ring has arcs composed of FODO cells, $9.8 \mathrm{~m}$ in length, which use $1-\mathrm{m}$ long quadrupoles of strength $45 \mathrm{~T} / \mathrm{m}$ and $2.4-\mathrm{m}$ long dipoles with a $6 \mathrm{~T}$ field.

Most recently, the calculations were performed for the Study-2 ring [2] for a muon beam momentum of $20 \mathrm{GeV} / \mathrm{c}$. There are $2 \times 10^{20}$ muons per year decaying in the $126-\mathrm{m}$ long production straight. The arcs are 53 meters for 180 degrees, i.e. $16.87-\mathrm{m}$ effective radius. Lattice functions with $60^{\circ}$ are shown in Fig. 2 [5]. These optics use skew quadrupole magnets for the main focusing so the the betatron eigenmodes lie along diagonal planes. This choice permits the use of a compact magnet focusing structure. But otherwise dispersion suppression, optics matching and chromaticity correction proceed as normal. The compact arc is important for a BNL site to where much of the ring will be above the existing ground level and will require construction of a covering hill. The BNL off-site limit is

\footnotetext{
* Work supported by the Universities Research Association, Inc., under contract DE-AC02-76CH03000 with the U. S. Department of Energy.

†mokhov@fnal.gov
}

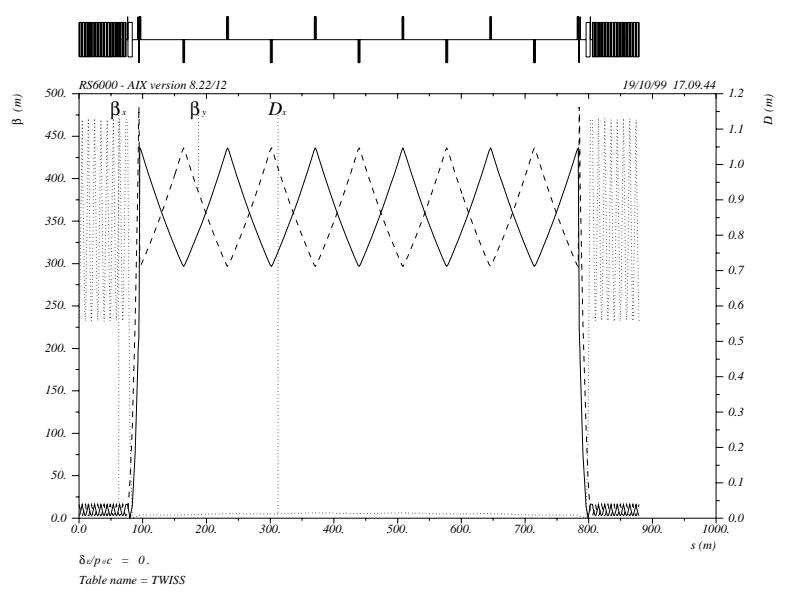

Figure 1: Lattice functions for the $50 \mathrm{GeV}$ half ring including the downward production straights.

$5 \mathrm{mrem} / \mathrm{yr}$, and the BNL drinking water standard requires less than $1 \mathrm{pCi} / \mathrm{ml}$ tritium and $0.2 \mathrm{pCi} / \mathrm{ml}{ }^{22} \mathrm{Na}$. For shielding calculations, wet soil properties were used with the density of $2.24 \mathrm{~g} / \mathrm{cm}^{3}$. For neutrino-induced radiation, the soil density is practically irrelevant, therefore the results are directly applicable to the BNL soil.

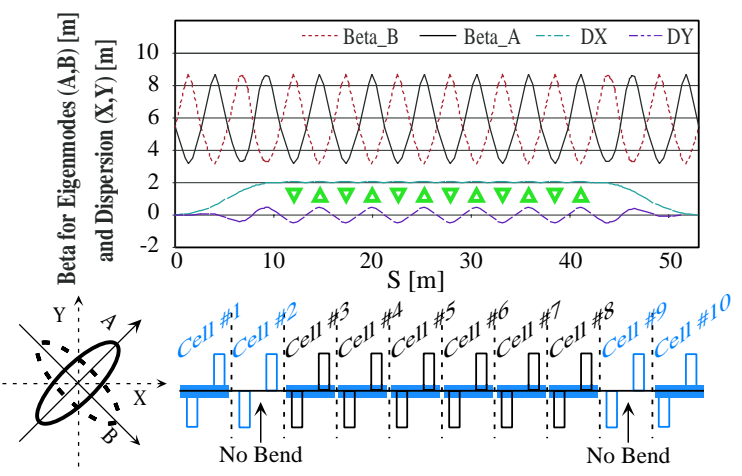

Figure 2: Eigenmode beta functions $(\mathrm{A}, \mathrm{B})$ and effective dispersion $(X, Y)$ plotted for a $20-\mathrm{GeV}$ muon storage ring arc. $(\mathrm{A}, \mathrm{B})$ and $(\mathrm{X}, \mathrm{Y})$ coordinate systems are shown.

\section{MAGNETS AND DYNAMIC LOSSES}

At $50 \mathrm{GeV}$, the muon decay rate is $2 \cdot 10^{12} \times 15 \times$ $165 \times 3.21 \cdot 10^{-6}=1.6 \cdot 10^{10}$ decays $/ \mathrm{m} / \mathrm{s}$. The beam's spatial and angular distributions are simulated using a rms transverse emittance of $3500 \pi \mathrm{mm}$-mrad and a rms $\Delta p / p$ of $1 \%$. Muon decay, $\mu \rightarrow e \nu \tilde{\nu}$, is characterized in the code using the exact decay kinematics. The decay electrons and, also, the synchrotron photons subsequently emitted by these electrons, eventually travel outside the machine aper- 
ture and induce electromagnetic showers which then propagate through the lattice elements. These showers are fully simulated with MARS 14 so that the corresponding heat load deposited in the magnets can be calculated along with the radiation fields generated in the tunnel and surrounding areas. Details of the power density distribution in the SC magnet components are essential to determining the quench stability of the magnets and the potential impact on the machine operation. The corresponding heat load delivered to the magnets also specifies the cooling requirements for the ring, both in water and cryogenic capability.

The magnet design proposed in Study-2 for the $20-\mathrm{GeV}$ ring has racetrack coils with an open mid-plane (Fig. 3) to keep decay products away from the superconducting coils [6].

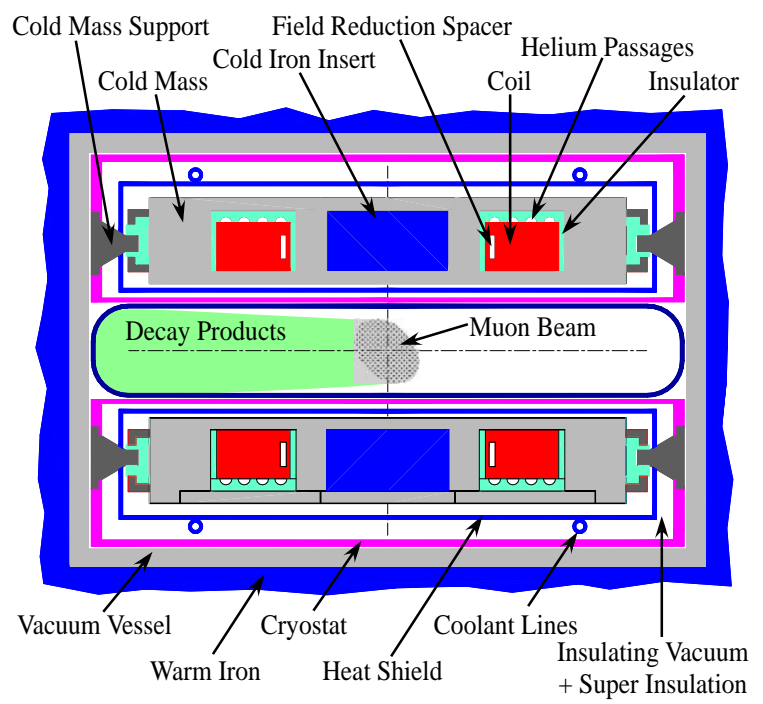

Figure 3: Cross section, with main features labeled, of the 20-GeV muon storage ring magnet.

\section{RADIATION IN ARC MAGNETS}

In Study-1 design with its cosine-theta magnet design, a $240-\mathrm{kW}$ circulating muon beam and a $1750-\mathrm{m}$ circumference, $84 \mathrm{~kW}$ of power is dissipated in the ring at $50 \mathrm{GeV}$, or 47.8 Watts per meter on average. Calculations show that most of this power must be intercepted by a tungsten tube inserted in the SC magnet bore. Longitudinal distribution of both power density and power dissipation oscillate periodically along the length of the arc cell. From Fig. 4, which is the case with bent dipoles (dipoles with a sagitta) and a 1-cm thick tungsten bore tube, it can be seen that $90 \%$ of the power is dissipated in the protective bore tube and only about $10 \%$ of power is dissipated in the SC coils. The power density peaks in the orbit plane exhibiting a strong azimuthal variation for the case with bent dipoles and an eccentric tungsten bore tube.

Table 1 compares the effect of a bore tube made of different materials. Due to the electromagnetic origin of energy deposition, the heavier material, the more localized the

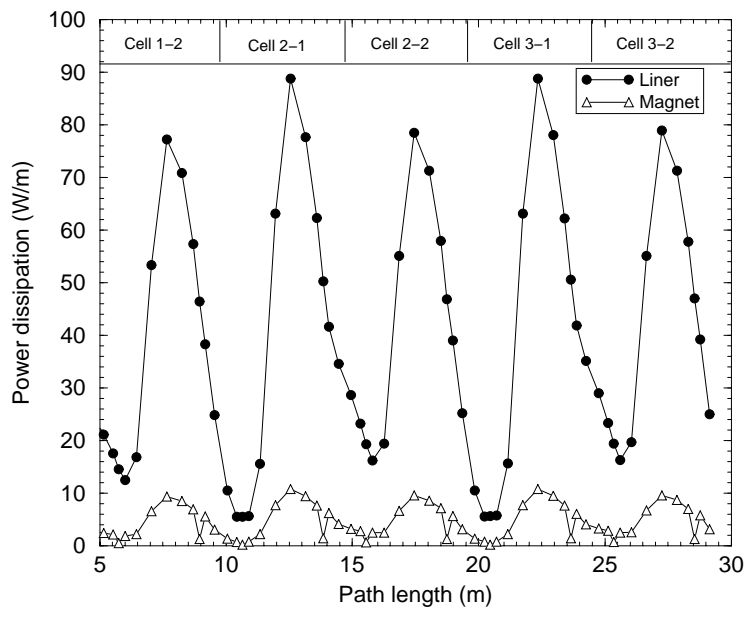

Figure 4: Power dissipation along the 50-GeV arc.

showers. With a tungsten liner, the induced activation and therefore the residual dose rates on the magnets meet the criteria for hands-on maintenance: $13,6,5$ and $3 \mu \mathrm{Sv} / \mathrm{hr}$ on liner, SC coil, He vessel and outer vessel, respectively, after 30 day irradiation and 1 day cooling.

Table 1: Power dissipation (W/m) averaged over $50-\mathrm{GeV}$ arc magnet components with a 1-cm thick liner made of tungsten, copper and aluminum: liner $(100 \times 80 \mathrm{~mm}$ ID), SS He tube $(65<\mathrm{r}<70 \mathrm{~mm})$, SC coil $(75<\mathrm{r}<95 \mathrm{~mm})$, rest of the magnet $(\mathrm{r}>95 \mathrm{~mm})$ and sum in the magnet $(\mathrm{r}>65 \mathrm{~mm})$.

\begin{tabular}{|c|c|c|c|c|c|}
\hline Mat. & Liner & Tube & Coil & Rest & $\mathrm{r}>65 \mathrm{~mm}$ \\
\hline $\mathrm{W}$ & 42.75 & 1.09 & 1.46 & 2.53 & 5.08 \\
$\mathrm{Cu}$ & 29.88 & 4.83 & 5.37 & 6.36 & 16.56 \\
$\mathrm{Al}$ & 5.25 & 11.63 & 15.08 & 13.94 & 40.65 \\
\hline
\end{tabular}

Peak power density in SC coils is noticeably higher in a straight dipole (Table 2, first line) compared to a bent one (rest of Table 2). Furthermore, since decay electrons are swept to the inward side of the dipole, thickening that side of the bore tube is particularly effective in reducing the power dissipated in the magnets. The combination of a bent dipole and an eccentric tungsten bore reduces peak power density in the SC coils (quench stability) and dynamic heat load to the arc magnets (cryogenic heat capacity) to the tolerable levels of $P_{\max } \leq 1 \mathrm{~mW} / \mathrm{g}$ and $\mathcal{P}_{\max } \leq 10 \mathrm{~W} / \mathrm{m}$, respectively (Table 2). An eccentric tungsten shield can be made of a $1-\mathrm{mm}$ thick stainless steel pipe $(100 \times 80 \mathrm{~mm}$ elliptical aperture) and a 1-mm thick stainless steel pipe $(122 \times 102 \mathrm{~mm}$ elliptical aperture) shifted inward by $5 \mathrm{~mm}$ with a space between filled with a tungsten-like material. With such a tube, the peak power density in the coil is only $0.15 \mathrm{~mW} / \mathrm{g}$, with the peak power dissipation in the bore tube (nitrogen temperature) and the rest of the magnet (helium temperature) about 90 and $9 \mathrm{~W} / \mathrm{m}$, respectively. Averaged over the arc length the values are approximately a factor of two lower than the peak. 
Table 2: Peak power density $P_{\max }(\mathrm{mW} / \mathrm{g})$ in SC coils and peak power dissipation $\mathcal{P}_{\max }(\mathrm{W} / \mathrm{m})$ in a bore tube and in the magnet $(\mathrm{r}>65 \mathrm{~mm})$ for a $50-\mathrm{GeV}$ arc.

\begin{tabular}{|l|l|c|c|}
\hline Bore tube & $P_{\max }$ & $\mathcal{P}_{\max }$ & $\mathcal{P}_{\max }$ \\
\hline & SC coil & Bore tube & Magnet \\
\hline $1 \mathrm{~cm} \mathrm{~W}$ & 0.424 & 109.7 & 13.87 \\
\hline $1 \mathrm{~cm} \mathrm{~W}$ & 0.285 & 88.75 & 10.84 \\
$1.5 \times 0.5 \mathrm{~W}$ & 0.147 & 90.45 & 8.875 \\
$1.5 \times 0.5 \mathrm{~Pb}$ & 0.352 & 81.92 & 16.68 \\
$1 \mathrm{~cm} \mathrm{Cu}$ & 2.790 & 54.61 & 40.11 \\
\hline
\end{tabular}

\section{RADIATION AROUND TUNNEL}

It is found that the normal occupancy limit of $0.25 \mathrm{mR} / \mathrm{hr}$ in the underground facilities (electronics rooms etc) is met with 2 to $2.5 \mathrm{~m}$ of dirt shielding below, above, and radially inward from the arc tunnel enclosure walls. Six to seven meters of such a shielding is needed to meet this limit radially outward from the ring, and 2 to $2.5 \mathrm{~m}$ around the straight tunnels. Conveniently, power supply rooms and other underground enclosures should be placed on the inward side of the arc tunnel permitting unlimited occupancy even during machine operation. For ground-water protection, there should be no water flow through the nearest to the tunnel $1.5-\mathrm{m}$ of soil in the above shielding.

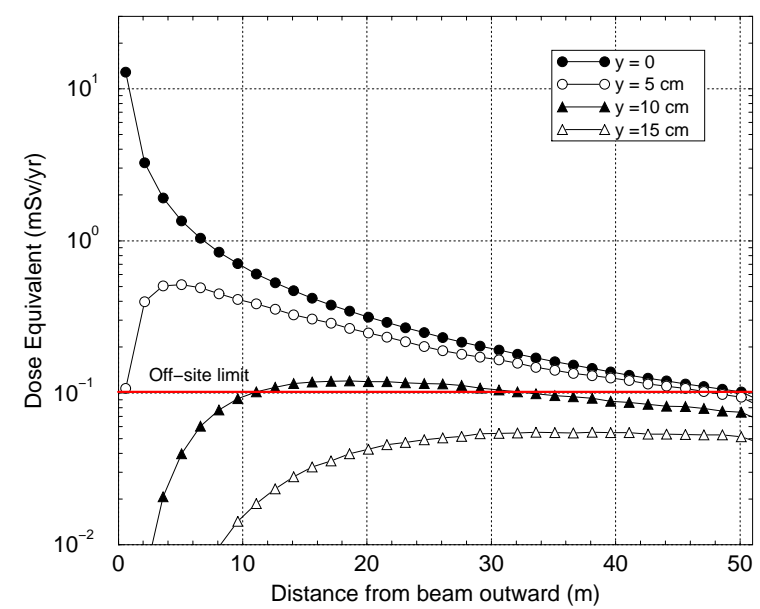

Figure 5: Neutrino-induced annual maximum dose equivalent in a phantom embedded in soil outward from a $50 \mathrm{GeV}$ arc tunnel at several heights with respect to the orbit plane.

The decay neutrinos are propagated through the dirt surrounding the tunnel, where they interact, and create daughter showers which produce radiation at very large distances [7]. The off-site limit of $10 \mathrm{mrem} / \mathrm{yr}$ or $0.1 \mathrm{mSv} / \mathrm{yr}$ in the Study-1 ring is reached at $50 \mathrm{~m}$ radially outward from the beam's orbit (Fig. 5), while in the Study-2 ring ( $5 \mathrm{mrem} / \mathrm{yr}$ ) it is reached at $30 \mathrm{~m}$ radially outward (Fig. 6). The height of the radiation disk reaches only $\pm 10 \mathrm{~cm}$ above and below the orbit plane.

Neutrino-induced radiation downstream of the straight sections is more severe than from the arcs. The off-site

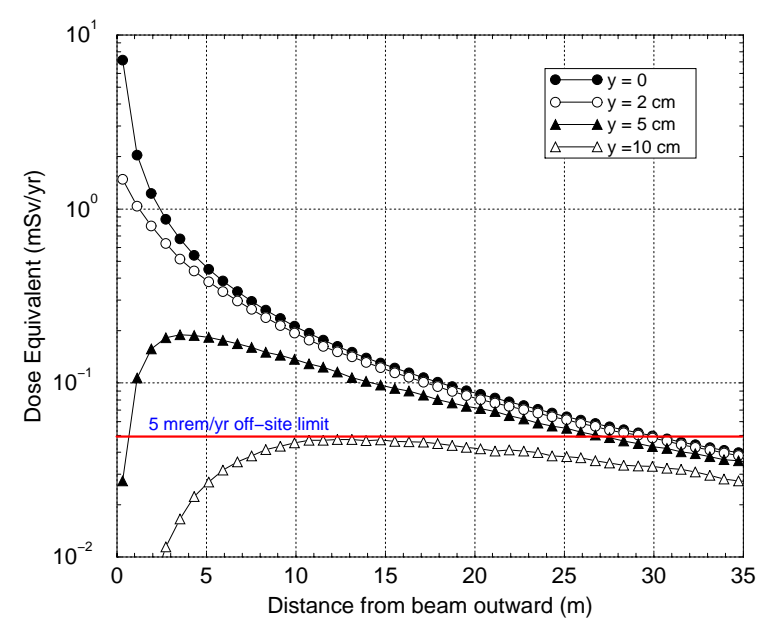

Figure 6: Same as in previous figure, for Study-2 design.

limits are only met after $4.2 \mathrm{~km}$ and $1.3 \mathrm{~km}$ for $50 \mathrm{GeV}$ and $20 \mathrm{GeV}$ muon storage rings, respectively. The maximum half-widths of an off-site limit isocontours are $4.3 \mathrm{~m}$ and $2 \mathrm{~m}$, respectively. For ground-water protection downstream the straight sections, one needs about $3 \mathrm{~m}$ immediately downstream. Neutrino-induced activation is below the BNL limits by a factor of 800 for tritium and even lower for ${ }^{22} \mathrm{Na}$ at maximum.

\section{CONCLUSIONS}

The radiation load to the SC coils of the arc magnets due to muon beam decays is reduced to the levels acceptable for quench stability by a tungsten eccentric bore tube (Study-1), and drastically minimized by choosing the open mid-plane magnet (Study-2). The shielding parameters found in this work ensure that both sites, Fermilab and BNL, are satisfactory for a Neutrino Factory from a radiation point of view.

\section{REFERENCES}

[1] N. Holtkamp and D. Finley, eds., A Feasibility Study of a Neutrino Source Based on a Muon Storage Ring, Fermilab-Pub00/108-E, 2000.

[2] http://www.cap.bnl.gov/mumu/studyii/FS2-report.html.

[3] N. V. Mokhov, “The MARS Code System User's Guide", Fermilab-FN-628 (1995); N. V. Mokhov and O. E. Krivosheev, "MARS Code Status", Fermilab-Conf00/181 (2000). http://www-ap.fnal.gov/MARS/.

[4] N.V. Mokhov, C.J. Johnstone, Neutrino Factory-2000, Monterey, CA, May 2000; Fermilab-Conf-00/207, 2000.

[5] B. Parker, "Skew Quadrupole Focusing Lattices and Applications", paper MOPA010 this conference.

[6] B. Parker, et.al., "Magnets for a Muon Storage Ring with Skew Focusing", paper RPPH073 this conference.

[7] N.V. Mokhov, A. Van Ginneken, in: Proc. of ICRS-9 International Conference on Radiation Shielding (Tsukuba, Ibaraki, Japan, 1999), J. Nucl. Sci. Tech. 1, 2000, pp. 172-179; Fermilab-Conf-00/065, 2000. 